EDUCATION

Research, Inovortion and Solutions on-line ${ }^{\oplus}$
PSYCHOLOGY

$1+D+i$
Electronic Journal of Research

in Educational Psychology

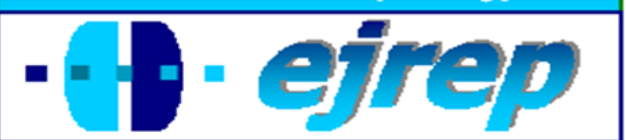

Editorial EOS

\title{
An Error Analysis of Form 2 \\ (Grade 7) Students in Simplifying Algebraic Expressions: A Descriptive Study
}

\section{Lim Kok Seng}

Faculty of Science and Technology, Open University Malaysia, Kuala Lumpur

\section{Malaysia}

Correspondence: Lim Kok Seng: Open University Malaysia, 86 Jalan Dato’ Bandar Tunggal 70000 Seremban, Negeri Sembilan, Malaysia. E-mail: ernie5287@yahoo.com

(C) Education \& Psychology $\mathrm{I}+\mathrm{D}+\mathrm{i}$ and Editorial EOS (Spain) 


\section{Abstract}

Introduction. This study aimed to investigate the errors made by 265 Form 2 male students in simplifying algebraic expressions.

Method. A total of 265 Form 2 (Grade 7) male students were selected for this study. 10 high, medium and low ability students in each group were selected for the interviews. 40 items were administered to the respondents to investigate their errors in simplifying algebraic expressions.

Results. This study identified 12 types of errors. A comparison of the types of errors exhibited by high, medium and low ability students was investigated.

Conclusión. All these errors might be the results of: (i) interference from new learning; (ii) difficulty in operating with the negative integers; (iii) misconceptions of algebraic expressions; and (iv) misapplication of rules.

Keywords. Systematic Error, Algebraic Expressions, Simplifying Algebraic Expressions, Bracket Expansion.

Received: 09/21/09 Initial Acceptance: 09/23/09 Definitive Acceptance: 11/25/09 


\section{Análisis de los errores de alumnos del primer curso de Educación Secundaria en la simplificación de expresiones algebraicas}

\section{Resumen}

Introducción. El estudio pretende investigar los errores cometidos por los alumnos de primer curso de Educación Secundaria en la simplificación de expresiones algebraicas.

Método. Un total de 265 alumnos del primer curso de Educación Secundaria fueron seleccionados para este estudio. Se seleccionaron, a través de entrevista, 10 alumnos de cada uno de los grupos de hablidad alta, habilidad media y habilidad baja. Se administró un instrumento con 40 ítems a los participantes para investigar los errorer en la simplificación de expresiones algebraicas.

Resultados. Este estudio identifica 12 tipos de errores. Se han comparado los errores cometidos por los estudiantes de habilidad alta, media y baja.

Conclusión. Todos los errores pueden ser resultado de: (i) intereferencia con el nuevo aprendizaje; (ii) dificultad para operar con enteros negatives; (iii) conceptos erróneos de expresiones algebraicas; y (iv) errores en la aplicación de reglas.

Palabras Clave. Error sistemático, expresiones algebraicas, simplificación de expresiones algebraicas, extensión de paréntesis.

Recibido: 21/09/09 Aceptación Inicial: 23/09/09 Aceptación Definitiva: 25/11/09 


\section{Introduction}

Algebra is an important topic in the school mathematics curriculum. The application of algebra can be found in all current branches of mathematics and science in general. In Malaysia, algebra covers one third of the secondary school mathematics curriculum (Ministry of Education Malaysia, 2003). It is considered an important topic in the PMR (Lower Secondary School Evaluation) and SPM (Malaysia Certificate of Education) examinations. Hence, proficiency in solving algebraic problems is pertinent to the students' overall mathematics achievement in the national examinations.

Research on the development of algebraic understanding by investigating the difficulties students encountered in solving algebraic problems has been well documented (Vlassis, 2002; Warren, 2003). However, most of these studies focused their attention on the topic of linear equations and the difficulties students encountered in solving linear equations. Comparatively, a few studies have been given to the topic of algebraic expressions (Ayres, 2000a; Demby, 1997; Liebenberg, 1997; Sakpakornkan \& Harries, 2003).

The 1999 Third International Mathematics and Science Study-Repeat report (Ministry of Education Malaysia, 2000) showed that less than half (46\%) of the Malaysian Form 2 students answered the three algebraic items correctly. Similarly, a survey of the 1994, 1995 and 1996 PMR Performance Reports (Ministry of Education Malaysia, 1994, 1995, 1996) showed that Form 3 (Grade 8) students did not perform well in simplifying items involving algebraic expressions. These students exhibited some learning difficulties in the topics of bracket expansion, exponent, negative integers, algebraic fractions and computations involving algebraic terms.

Corder (1982) proposed two justifications for the study of learners' errors: the pedagogical justification and the theoretical justification. Theoretical justification claims that learners' error helps us understand their learning process. Therefore, error identification can be a useful tool for researchers to investigate the mechanisms used by students in obtaining, processing, retaining, and reproducing the information contained in mathematical tasks. 


\section{The misconceptions in algebra}

Algebra involves variables; whereas algebraic expressions contains variables, constants amd operational signs. Hence, students are required to understand the concepts of variables and the meanings of algebraic terms in order to reduce an algebraic expressions correctly (Filloy \& Rojano, 1989). Research studies (Herscovics \& Linchevski, 1994; Kieran, 1997; Boulton-Lewis \& et. al., 2000; Radford, 2000; Hall, 2002a; Vlassis, 2002) had identified a number of errors and misconceptions on students' understanding of algebra.

According to Bodin and Capponi (1996), the concepts of variables vary according to the topic of problem that the students have to handle. The idea that a variable not only represents a particular value but can represent different values such as in the case of $x+y$ (Filloy $\&$ Rojano, 1989). Sfard and Linchevski's (1994) theory defines the algebraic expressions concepts in two different ways: operationally and structurally. These dual interpretations of algebraic expressions create serious difficulties for many students.

The arithmetic-algebra gap remains a fundamental cause of learning difficulties, because knowledge of algebra is built up on the foundation of already acquired arithmetical knowledge (Bodin \& Capponi, 1996). Arithmetic is concerned with the solving of numerical problems and in essence uses the whole natural numbers and positive decimals. Algebra is linked to the use of symbolic representations to express the variables and possibility of calculating on the basic of expressions involving the symbolic representations (Filloy \& Rojano, 1989).

Warren (2003) felt that understandings of basic arithmetic operations could assist successful transition from arithmetic to algebra. Her findings suggest that understanding of mathematical notion and arithmetic operations are basic criteria for the success in acquisition of algebraic knowledge. Pimm (1995) claimed that fluent users are aware that there are two differences when working with algebraic expressions: being able to see them as structured strings of symbols and as descriptions connected with some situation.

Students use the equals sign in both contexts: arithmetic and algebra. The concept of the equals sign in the framework of arithmetic is that of a 'do something' signal (Bodin \& Capponi, 1996). Many students tried to add " $=0 "$ to algebraic expressions when they were 
asked to simplify (Kieran, 1997). Tirosh, Even and Robinson (1998) point out that the procedural thinking students frequently face difficulties accepting 'lack of closure' solution. They think that open expression as incomplete and tend to find the final answer.

Many children do not understand the idea of letter as a variable (Booth, 1981). They tend to interpret letter as standing for a specific number, and different letters must necessarily represent different numbers (Collis \& Romberg, 1975; Kuchemann, 1981). Kuchemann (1981) described the students' views of algebraic symbols in terms of hierarchical levels, namely, letter ignored, letter is evaluated, letter as object, letter as specific unknown, letter as generalized number and letter as variable. Children are reluctant to record an algebraic statement as an answer; they tend to give numerical values to letters or variables (Collis \& Romberg, 1975; Kuchemann, 1981).

Likewise, many students ignore the proper use of brackets, assuming that the context of the problem determines the order of operations. Their conventional absence in multiplication due to priority, raise difficulties to the students. The conventional operations seem useless to students at the beginning of algebra.

The terminology and the rules of algebra that are obvious to teachers may be a source of confusion to children. Many of them have difficulties in trying to keep track of abstract terminology and to manipulate symbols and numerals simultaneously. Many rules of algebra seem arbitrary and meaningless to children, and this often lead them to invent their own malrules (Demby, 1997). This occurs when some prototype rules are created by generalizing taught systematic rules (Demby, 1997; Radatz, 1979).

\section{Errors in solving algebraic problems}

Students made all kinds of computational errors in solving mathematical problems. Computational errors are not necessary the result of carelessness or not knowing how to proceed, it can be caused by students applying the 'failure strategies' (Ashlock, 1994). Research into errors provides a rich source of knowledge about the processes and influences involved in mathematical problem solving. Considerable research has been conducted into the nature and causes of errors in solving algebraic problems. 
Clement (1982) reported that a large number of science-oriented college students were unable to solve a very simple kind of algebra word problem. These errors were from two main sources: syntactic and semantic. Loh (1991) studied the levels of ability in solving algebra word problems among 130 secondary two students. The results indicated that problem understanding and problem representation are the critical rate-determining steps in solving algebra word problems.

Hall (2002a) investigated the errors made by 246 pupils in solving three to six linear equation questions. Transposing, switching addends and division are found to account for approximately three quarters of the total number of errors. Ayres (2000b) identified the errors committed by students on bracket expansion tasks as working memory load was not equally distributed over the four operations.

Liebenberg (1997) designed a diagnostic test to investigate the errors made by 40 students in simplifying very elementary algebraic expressions. Liebenberg reported that three error patterns occurred in his study, namely misinterpretation of symbolic notation, difficulty with the subtraction concept, and difficulty with the integers.

Sakpakornkan and Harries (2003) explored pupils' processes of thinking in simplifying algebraic items. The main difficulties were dealing with negative signs and multiplying out the brackets. Similarly, Demby (1997) focused on the types of procedures used by students in performing the task. Most of the procedures appeared spontaneous in the sense that they had not been taught in the class.

\section{The Theoretical Framework}

According to Piaget (1970), individual thoughts during the process of learning consist of two basic alternative mechanisms: accommodation and assimilation. Piaget identified four main stages of development, namely sensori-motor stage, pre-operational stage, concrete operations stage and formal operations stage. Piaget uses the words accommodation and assimilation to describe individual adjusting the mind to new experience and the process by which individual takes new data. Piaget's idea of mathematical processes and mental concepts has become basis for many theories. 
Gray and Tall (1994) see the mathematical symbol as pivot between processes and concept. Their research focuses on the differences between procedural and proceptual thinking. An algebraic expression such as " $2 a+5 b$ " is a procept which stand dually for the process add two times $a$ to five times $b$ which can be manipulated mentally as an object.

Mathematical procedures can cause problems in a variety of ways. Mathematical rules remain unchanged and being used inappropriately, new rules may be used incorrectly. For instance, students conceive the expressions $4+3 x$ as mental objects, error occurs if students are unable to accept such expressions as an answer. Procedural approach students see algebraic expressions as a process by finding its solution. A mathematical idea is learnt in a meaningful way in one context, this may cause conceptual difficulties when the old meanings no longer hold in a new context. For example, bracket means priority of computing in arithmetic. However, this conventional operation can not be applied in algebraic expressions.

Students using alternatives ways to solve problems show greater flexibility and efficiency in solving a given question. The ability to think that symbols as an entity allows individuals to move between process and concept. This means that those who are focused mainly on the procedural have difficulties in learning new mathematics compared to those who are able to focus on the symbolism as both process and concept. Procedural students are encouraged to move towards a proceptual approach once they have learned the topic of algebra.

\section{Method}

Students' thinking processes and procedures used in simplifying these items were obtained through their answer scripts and interview transcripts. Students used appropriate or inappropriate procedures in simplifying algebraic expressions. Their underlying concepts were revealed through execution of these procedures. The errors made by students were classified according to the types of errors and quantified according to their frequencies of occurrences. These classification and quantification of errors provided an opportunity for the researcher to discover the errors made by low, medium and high ability students and the possible causes of these errors by analyzing in detail the inappropriate procedures being used by the students. Interviews were conducted to justify the procedures used in obtaining their solutions. Interviews provided an insight into students' thinking processes that are not usually in their working. 


\section{Participants}

The respondents of this study comprised of 265 Form 2 male students from a secondary school whose average age was 14 years. The students were divided into three different ability groups based to the results in their Form 1 school mathematics final assessments. 10 low, 10 medium and 10 high ability students were chosen for the interviews based on patterns of errors they had employed in the algebraic expressions tests.

\section{Instruments}

In this study, a set of 32 open-ended algebraic expressions items was constructed for the preliminary study. All these items were adopted and adapted from various educational studies on this topic (e. g. Ayres, 2000b; Booth, 1981; Demby, 1997; Hart et. al., 1981; Liebenberg, 1997; Sakpakornkan \& Harries, 2003; Swafford \& Brown, 1989; Teng, 2002; Vaiyavutjamai, 2001; Vlassis, 2002). These 32 algebraic expressions items were administered to 38 Form 2 students in another school. A preliminary study had provided the researcher to examine the types of errors appeared in students' answer scripts.

From the preliminary study, the lowest scores for each item in each level were identified. Analysis of these students' errors revealed that 11 types of errors made by students in their written solutions. The most prevalent error made by students was incorrect order of operations, followed by misinterpretation of symbolic notation error, negative pre-multiplier error and addition of integers error. Other errors made by students in the preliminary study involved the operations of variables, coefficients of 1 or -1 , brackets expansion tasks, negative integers, negative pre-multiplier, like terms in the form of $a^{2}$ and $a b$ and the application of BODMAS (order of operations - Brackets, Orders, Division and Multiplication, Addition and Subtraction) rule in algebra.

One item from each level with the lowest number of correct responses was analyzed in detail. The frequencies of incorrect responses were identified and the types of errors were classified according to the characteristics of these errors. For instance, 25 students used the 'left-to-right' order method to simplify $a+a+2 a x 5$ in preliminary study. Therefore, six items included two expanded items were designed based on students' knowledge of BODMAS rule. 
A total of 40 algebraic expressions items were developed from the preliminary study. The computations involved were addition, subtraction and multiplication. These items were divided into (a) like terms and unlike terms; (b) integers; (c) simple algebraic expressions; (d) multiplication of variables; (e) brackets expansion; and (f) complex algebraic expressions.

\section{Procedure}

According to the NCTM Standards (2000), assessment of students' knowledge of procedures should determine not only whether student can execute procedures, but also whether they know the underlying concepts, when to apply the procedures, why the procedure work, and how to verify that the procedures yield correct answers. Therefore, all the respondents were requested to show their solution processes on the space provided in the instruments.

The interviews were carried out over three weeks during school by the researcher. The thirty students chosen for the interviews were selected on the basis of their Form 1 mathematics final assessment. Ten low, ten medium and ten high ability students were called for the interviews based on the systematic errors they made in their answer scripts. An audio recording was used to collect more detailed information for later analysis. With audio taping, various patterns, procedures and errors could be identified and transcribed. This technique should be used when we want to probe more deeply into a student's thinking processes, problemsolving performance, or attitudes.

The students were asked a sequence of questions by the researcher. These questions would reveal students' thinking as well as explaining and justifying the procedures and strategies used in simplifying algebraic expressions.

\section{Data Analysis}

Error analysis has been used widely by researchers as a means of making inferences about the nature of mental processes in mathematical thinking. Mulhern (1989) suggests four types of approaches to the study of errors in mathematics: (1) counting of the number of incorrect solutions; (2) analysis of the types of error; (3) analysis of error patterns; and (4) constructing problems in such a way as to induce errors in individuals. 
The process of analysis consisted of the following steps: identification of errors, description of errors, classification of errors, quantification of errors, and making inference of causes of errors. This analysis of students' works focused on procedural and conceptual errors. Procedural errors primarily consisted of using incorrect mathematical operations. Conceptual errors included selecting inappropriate model and application of algebraic principles.

Quantitative process is to identify the similarities and differences in errors made by high, medium and low ability students. This process of data provided a link to the error patterns made by high, medium and low ability students. This approach of analysis led to the causes of errors where remedial and diagnostic work could be recommended.

The audio-taping was employed during interviews. The audio-taped sessions were transcribed and categorized according to high, medium, and low ability students. These data were analyzed at two purposes, a) to identify the causes of errors and b) to triangulate against students' errors by constructing new problems to induce similar errors.

\section{Results}

\section{2 types of error made by Form 2 students when simplifying algebraic expressions}

There were three types of exponent errors made by students when simplifying algebraic expressions algebraic expressions involving multiplication and complex algebraic expressions. These errors occurred because students misinterpreted the exponent of variables. (i) Students added the exponents of the variables in simple algebraic expressions; (ii) Students misinterpreted the exponent of ' 2 ' in the variable $a^{2}$ by thinking that $3 a^{2}$ was equivalent to $3^{2} a$; and (iii) Four students had simplified $3 a^{2}+4 a^{2}$ as $7 a^{2}$ successfully, but wrote their final answer as $14 a$.

Students made more detachment from the negative sign errors in complex algebraic expressions compared to simple algebraic expressions. Students made two types of detachment from the negative sign errors in simplifying algebraic expressions. (i) Students simplified $-6 a+3 a$ as $-9 a$. Students performed the operations of addition without considering the 
negative sign attached to its integer. They then put the negative sign back in their solutions; and (ii) Detachment from the negative sign error can be identified in complex algebraic expressions.

For complex algebraic expressions, students simplified 5ab-6+4ba+7 (item 37) as $9 a b-13$. They added the integers in these algebraic terms and put their negative sign back in their solutions. In this study, students solved integer item -6+7 (item 6) correctly but made detachment from the negative sign error when $-6+7$ appears in the middle of an expression.

Misinterpretation of symbolic notation error occurred in items involving the variables of $a, b$ and $a^{2}$. The 'invisible' numerical coefficients of 1 in $a, b$ and $a^{2}$, led to its interpretation as ' 0 ' $a$, ' 0 ' $b$ and ' 0 ' $a^{2}$. For example, a student showed his working for $-8 a-a$ as $+8 a$. The students who made this type of error said: "nothing in front of $a$ ". They obtained the positive solution from the above item by using one of the multiplication rules: "negative times negative is positive".

Conjoin error appeared in simple algebraic expressions, bracket expansion and students' final answers. Two types of conjoin errors occurred in this study. (i) Students conjoined $4 a+3$ as $7 a$ in simple algebraic expressions; and (ii) Low and medium ability students tended to make conjoin error in bracket expansion. For instance, students simplified $2(4 a+3)=2(7 a)$. Students conjoined the algebraic terms in the bracket by misapplying the BODMAS rule of arithmetic in algebra.

Subtraction of integers error occurred more frequently in solving integer items and simple algebraic expressions. There are two types of subtraction of integers errors in students' answer scripts. (i) A student simplified $3 a-6 a$ by saying: "I take $6 a$ minus $3 a$, so I got $3 a$ ". Evidence from the students' interviews demonstrates that some students simplified the items by using the concept of subtraction in arithmetic "larger value minus smaller value"; and (ii) Students misapplied the rule "negative times negative is positive" in simplifying algebraic expressions involving two successive “-” signs. It appeared as if addition was carried out and the multiplication rule for integers applied. This kind of error was also evident in the language that students used during interviews. 
Two types of addition of integers errors occurred in this study. (i) Students simplified $-6 a+3 a$ as $3 a$ in simple algebraic expressions. The ' + ' sign seemed to lose its role as an operation sign in this item; and (ii) Students made addition of integers errors in $5 a b-6+4 b a+7$ (item 37) as 9ab-1. During interviews, students used the procedural approach rather than number line approach in the addition and subtraction of integer.

Students tended to make distributive error in bracket expansion. There are two types of distributive errors in this study. (i) Students simplified $2(4 a+3)$ as $8 a+3$. They multiplied the first algebraic term and ignored the second algebraic term in the bracket; and (ii) Students made errors in items involving one pre-multiplier such as $2(3 a+2)+(3+4 a)$. They multiplied the second bracket with pre-multiplier 2 and obtained $6 a+4+6+8 a$.

Two types of multiplication of variables error occurred in simplifying algebraic expressions involving multiplication. Students made errors when multiplied two variables or multiplied a variable and a number. (i) Students applied the rules of addition or multiplication in simplifying $a \times a$ and $2 a \times a$. They either multiplied or added the coefficients of $a$ and copied the variables without change; and (ii) Students made multiplication of variables error in items involving numbers and variables. For instance, students simplified $2 \times a+a+15$ as $30+a+a$

Many students (68.9\%) had difficulties to simplify $a+2 a \times 5$. They tended to simplify this item from 'left-to-right' order. This study shows that incorrect order of operations error occurred more frequently if the addition sign appeared before the multiplication sign.

Students understood the differences between like and unlike terms by analyzing their answers in items 1 to 5 . Some students had a misconception about like terms. They thought that $5 a b$ and $4 b a$ were unlike terms because they didn't understand the concept of like terms and the basic properties of commutative law.

Negative pre-multiplier error occurred when students did not change the sign of the second algebraic term after multiplying out the brackets. For instance, students simplified $3(6 a-7)$ as $-18 a-21$. 
Basically, students made careless error when they multiplied two integers. This error occurred because students were unfamiliar with the tables of multiplication.

\section{Differences in the errors made by high, medium and low ability students}

\section{Like term}

The percentages of errors for item 3 (-3cd and 10dc) were the highest among high, medium and low ability students $(\mathrm{H}=17.1 \%, \mathrm{M}=42.9 \%$ and $\mathrm{L}=71.8 \%)$. Students were confused between the concept of negative integers and the concept of like terms. High and medium ability students made a few errors in recognizing like and unlike terms. In contrast, $45.9 \%$ or 39 of the low ability students made errors in item 2 ( $4 b a$ and $5 a b)$ and item $4\left(3 a^{2} b\right.$ and $\left.3 a b^{2}\right)$ as $7 a^{2}$ respectively. Evidence from the interview transcripts revealed that low ability students did not understand the concept of like terms. These students had misconceptions of like terms by comparing their coefficients instead of their variables.

\section{Integers}

Of the seven items on integers (items 6 to 12), four types of errors were identified. The errors were detachment from the negative sign error, subtraction of integers error, addition of integers error and incorrect order of operations error. High ability students demonstrated the least errors in solving all the integers items. High ability students made more errors in addition of integers (35.3\%) and incorrect order of operations (29.4\%) compared to other items. In contrast, medium and low ability students made more detachment from the negative sign errors $(\mathrm{M}=25.8 \%$ and $\mathrm{L}=38.6 \%)$ and subtraction of integers errors $(\mathrm{M}=39.4 \%$ and $\mathrm{L}=32.9 \%)$ in solving integers items.

\section{Simple algebraic expressions}

There were seven types of errors made by the high, medium and low ability students in simplifying simple algebraic expressions. Exponent error $(\mathrm{H}=46.8 \%, \mathrm{M}=42.9$ and $\mathrm{L}=37.5 \%$ ) was the highest compared to all other types of errors in these three different ability students. Exponent error occurred more frequently in item $19\left(3 a^{2}+4 a^{2}\right)$. Conjoin error was the second highest number of errors $(21.3 \%)$ made by high ability students. 
Besides exponent error, $19.6 \%$ or 44 medium ability students conjoined item 14 $(4 a+3)$ as $7 a$ in their final answers. The third and fourth highest numbers of errors made by medium ability students were subtraction of integers errors (13.8\%) and detachment from the negative sign error (12.5\%). The second highest number of errors made by low ability students in simple algebraic expressions was the misinterpretation of symbolic notation $(18.5 \%)$, followed by conjoin errors (16.1\%) and subtraction of integers errors (13.9\%).

\section{Algebraic expression involving multiplication}

Five types of errors were identified in simplifying algebraic expressions involving multiplication. Analysis of students' errors indicated that high and low ability students exhibited the highest percentages of multiplication of variables errors $(\mathrm{H}=47.4 \%$ and $\mathrm{L}=48.4 \%)$ in simplifying these items. The second highest number of errors made by these two groups of students was of incorrect order of operation errors $(\mathrm{H}=31.0 \%$ and $\mathrm{L}=36.6 \%)$ when simplifying items 24 to 27 .

Medium ability students exhibited $48.5 \%$ of incorrect order of operations errors in simplifying items 24 to 27 . The second highest percentage of error (35.1\%) made by medium ability students was multiplication of variables error. Conjoin errors occurred more frequently in the final answers of high and medium ability students.

\section{Bracket expansion}

High ability students had difficulties in operating with the negative pre-multiplier. They made the highest percentage of negative pre-multiplier errors $(40.2 \%)$ in items involving bracket expansion. This group of student also made $27.7 \%$ of distributive errors in these five items.

Conjoin error was the most prevalent error among medium and low ability students in simplifying bracket expansion (43.1\% and 62.8\%). These two groups of students tended to conjoin the algebraic terms in the brackets before multiplying them with their pre-multipliers. $35.1 \%$ of medium ability students did not understand the distributive law in algebraic expressions.

The third highest percentage of error made by medium ability students was negative pre-multiplier error (15.2\%). Medium ability students only multiplied the algebraic terms 
within the brackets by its pre-multiplier by not considering the negative sign attached to its pre-multiplier. Besides conjoin error, low ability students made $22.1 \%$ and $15.0 \%$ of distributive error and negative pre-multiplier error respectively in simplifying bracket expansion. They made only three types of errors because 23 out of 63 students either left their answers blank or wrote incomplete answers for these five items.

\section{Complex algebraic expressions}

Detachment from the negative sign error was the most dominating errors exhibited by these three groups of students $(\mathrm{H}=34.2 \%, \mathrm{M}=38.7 \%$ and $\mathrm{L}=33.3 \%)$. Besides, high ability students tended to make addition of integers error (21.9\%) in simplifying complex algebraic expansion. This group of students also weak in operating symbolic notation $a, b$ and $a^{2}(15.1 \%)$.

Medium ability students made the most number of detachment from the negative sign errors (38.7\%) in simplifying item 33 to item 40. This error occurred more frequently in item 37 compared to other items. The second highest number of errors made by medium ability students was exponent errors (22.6\%), followed by misinterpretation of symbolic notation errors $(16.1 \%)$.

Low ability students made a total of 54 errors only as 27 of these students either left their answers blank or gave incomplete responses in their answer scripts when simplifying item 33 to item 40. Low ability students made $33.3 \%$ of detachment from the negative sign errors and $25.9 \%$ exponent errors in simplifying these items. They tended to conjoin all the algebraic terms and exhibited subtraction of integers errors (13.0\%) in simplifying complex algebraic expressions.

\section{Discussion and Conclusions}

\section{Interference of new learning}

The outcomes of this study suggest that, exponent error is the prevalent error made by Form 2 students in simplifying algebraic expressions. Analysis of students' errors shows that 
Form 2 students tended to make the exponent error because of interference from the newly learned mathematical concepts. This is evidenced in the interview transcripts with students.

MacGregor \& Stacey (1997) claimed that older students had more opportunities for making exponent errors because of interference of new learning. When algebraic concepts are not used in other parts of the mathematics curriculum, students forget the notation for expressing them. Consequently, when new concepts and notations are introduced, students are unable to differentiate them from what they have previously been taught.

\section{Difficulty in operating with the negative integers}

Analysis of errors made by Form 2 students in integers (items 6 to 12) indicated that students had difficulties in manipulating integers, which was a subordinate skill necessary in the solution of algebraic expressions. They made more errors when negative integers appeared as coefficients in algebraic expressions. These errors occurred because students misapplied rules that they had learned in arithmetic. Students had a tendency to use the procedural approaches in manipulating negative integers.

Students made detachment from the negative sign error thought that $-6 x+3 x$ could be solve as $-(6 x+3 x)$. Consequently, they obtained the incorrect answer $-9 a$. This finding concurs with Hall's (2002b) study on 246 students with ages ranging from twelve to sixteen years that students had a tendency to ignore the negative sign preceding a number.

Students also made detachment from the negative sign errors in complex algebraic expressions. Form 2 students had a tendency to divide complex algebraic expressions into different entities before simplified it. Vlassis (2002) claimed that $8^{\text {th }}$ grade students made detachment from the negative sign errors when the negative signs appeared in the middle of expressions.

A considerable number of students made ' + ' and '- ' signs errors when multiplied out negative pre-multipliers in bracket expansion. Students applied the distributive law correctly but made '+' or '-' sign error in the second algebraic term. The possible cause of this error is students multiplied the pre-multiplier without considered the negative sign attached to its premultiplier. 
Studies have shown that many students have great difficulties in understanding the basic concepts of algebra (Herscovics \& Linchevski, 1995; Kuchemenn, 1981; MacGregor \& Stacey, 1996). In this study, the misinterpretation of symbolic notation errors occurred because students had misconceptions of algebraic terms $a, b$ and $a^{2}$.

One of the underlying causes of this error is the appearance of the 'invisible' coefficients in front of these variables. Students tended to ignore the numerical coefficient of 1 attached to $a$. Conjoin error occurred because students did not understand the concept of algebraic expressions. They conjoined a variable and a number in an algebraic expression. Gray and Tall (1994) observed that the lack of formation of the procept for algebraic expressions caused difficulties to the students. Students simplified $4 a+3$ as $7 a$ as they see the symbolism representing only a general procedure for computation.

Many medium and low ability students (41.4\% and 59.5\%) did not understand the concept of bracket in algebraic expressions by conjoining the algebraic terms in the brackets. Boulton-Lewis, Cooper, Atweh, Pillay and Wilss (2000) noted that the knowledge of distributive property is essential when students expand brackets. Students made conjoin errors in bracket expansion because they did not view algebraic terms in the bracket as an entity by itself.

A number of students in this study were unable to recognize like terms in the forms of $a b$ and $b a$. The underlying cause for this error is the concept of like terms and the law of commutative.

\section{Misapplication of algebraic rules}

Students misapplied a wide variety of rules in the process of simplifying these items. For instance, students misapplied the distributive law by multiplying only one of the algebraic terms in the bracket. Many students see bracket as priority of operations. Therefore, procedural approach students tended to conjoin the algebraic terms in bracket before added or multiplied it by its pre-multiplier. 
Misapplication of rules could be found in the multiplication of variables. For $a \times a$, students added or multiplied the coefficients of $a$ by obtaining $2 a$ or $a$ as their incorrect answers. Similarly, students multiplied 2 to 15 in item $2 \times a+a+15$ because they considered numbers were like terms in this item.

Incorrect order of operations errors occurred because students did not apply the BODMAS rule in algebraic expressions involving multiplication. Kieran (1979) pinpointed that the possible cause of this error is the lack of underlying knowledge of the structure of arithmetic operations.

The findings show that students in this sample make more exponent errors, misinterpretation of symbolic notation errors and conjoin errors and subtraction of integer errors in simplifying simple algebraic expressions items. Exponent error occurred because students were confused between the concepts of multiplication and addition of variables. One of the possible causes for this error is students try using the new algebraic concepts that they have learned to simplify some of the items in algebraic expressions. Students made misinterpretation of symbolic notation errors when the 'invisible' coefficient of 1 appeared in an expression. Some students had misconceptions of variables such as $a, b$ and $a^{2}$. Students conjoined a variable and a number as they were unwilling to accept the 'lack of closure' solutions. This indicates that students did not understand the underlying concept of algebraic expressions. Subtraction of the integers error occurred as students had difficulties in operating with negative integers.

In simplifying algebraic expressions involving multiplication, students made two main types of errors: multiplication of variables error and incorrect order of operation error. Students made multiplication of variables errors as they misapplied the rules of addition in the multiplication of variables. Incorrect order of operations error occurred as students simplified algebraic expressions from the 'left-to-right' order without considering the priority of operations in arithmetic (BODMAS rule).

Students tended to make conjoin error, distributive error and negative pre-multiplier error in simplifying bracket expansion. A considerable number of low and medium ability students thought that brackets meant priority of operations. Therefore, they conjoined the algebraic terms in the bracket. The distributive errors occurred as students misapplied the dis- 
tributive rule by just multiplying one of the algebraic terms in bracket. The presence of negative pre-multiplier in bracket expansion also causes many errors. Some students multiplied the pre-multiplier by not considering the negative sign attached to its pre-multiplier.

However, there was only one main type of error made by students in simplifying complex algebraic expressions. The most prevalent error was the detachment from the negative sign error. This difficulty appeared as students' lack of understanding of the concepts involved in the algebraic operations and symbolism. The students were not aware of the duality of the minus sign in algebraic expressions.

Generally, high ability students tended to make exponent error in simple algebraic expressions. They also encountered difficulties in the algebraic expressions involving multiplication and made errors when dealing with negative pre-multiplier in bracket expansion. Detachment from the negative sign error occurred only in complex algebraic expressions.

Medium ability students made the highest number of exponent errors in simple algebraic expressions. They conjoined a variable and a number in simple algebraic expressions as they see symbolism as general procedure for computation. Medium ability students made multiplication of variables errors and incorrect order of operations errors in simplifying algebraic expressions involving multiplication. These students either conjoined the algebraic terms in bracket or misapplied the distributive law in bracket expansion. Like the high ability students, they made detachment from the negative sign error mostly in complex algebraic expressions.

Low ability students made many different types of errors in simple algebraic expressions. They tended to make exponent error, misinterpretation of symbolic notation error and conjoin error in simple algebraic expressions. Besides, many low ability students had difficulties in operating with negative integers. They were confused between the multiplication and the addition of variables and did not apply the BODMAS rule when simplified algebraic expressions involving multiplication. Low ability students conjoined the algebraic terms in bracket as they did not understand the function of bracket in algebraic expressions. The findings show that they were weak in using some of the basic mathematical and algebraic skills in simplifying algebraic expressions. 
This study identified 12 types of errors in simplifying algebraic expressions. A comparison of the types of errors exhibited by high, medium and low ability students was investigated. All these errors might be the results of: (i) interference from new learning; (ii) difficulty in operating with the negative integers; (iii) misconceptions of algebraic expressions; and (iv) misapplication of rules. Moreover, the findings of this study highlighted two issues for mathematics teachers to consider when teaching algebraic expressions. The study revealed that many students still lack a good conceptual understanding of algebraic expressions. Hence, the findings implies that to enhance the quality of teaching algebraic expressions, the teacher need to explain the concept of algebraic expressions fully rather than just teach them the procedures for simplifying algebraic expressions. Form 2 students need to revise repeatedly the old algebraic rules or operations that they have learned in Form 1 in order to overcome the confusion between operations. However, students must be able to apply the rules and understand the logical processes in algebraic operations. Lastly, the instrument developed in this study can serve as a useful tool to diagnose and evaluate students' conceptual understanding of algebraic expressions.

\section{References}

Ashlock, R. B. (1994). Error patterns in computation (6 ${ }^{\text {th }}$ ed.). New York: Macmillan. Ayres, P. (2000a). Mental effort and errors in bracket expansion tasks. In J. Bana, \& A. Chapman (Eds.). Mathematics education beyond 2000 (80-86). Australia: MERGA 23.

Ayres, P. (2000b). An analysis of bracket exapsion errors. In Tadao Nakahara and Masataka Koyama (Eds.), Proceedings of the $24^{\text {th }}$ conference of the International Group of the psychology of Mathematics Education Vol. 4 (pp. 23-27). Hirishima, Japan

Bodin, A., \& Capponi B. (1996). Junior secondary school practices. In A. J. Bishop et. al. (Eds.), International Handbook of Mathematics Education, (565-614). The Netherlands: Kluwer Academic Publishers.

Booth, L.R (1981). Child-methods in secondary mathematics. Educational Studies in Mathematics, 12, 29-41.

Boulton-Lewis, G.M., Cooper, T.J., Atweh, B., Pillay, H., \& Wilss, L. (2000). Readiness for algebra. In Tadao Nakahara and Masataka Koyama (Eds.), Proceedings of the $24^{\text {th }}$ conference of the International Group of the psychology of Mathematics Education Vol. 4 (pp. 89-96). Hirishima, Japan 
Clement, J. (1982). Algebra word problem solutions: Thought process underlying a common misconception. Journal for Research in Mathematics Education, 13(4), 16-30.

Collis. K. \& Romberg, T.A. (1991). Assesment of Mathematical Performance: An analysis of open-ended Test items. In M. C. Wittroch, \& E.L. Baker, (Eds.), Testing and cognition (82-128). Engelwood Cliffs, NJ: Prentice Hall

Corder S.P. (1982). Error Analysis \& interlanguage. Oxford: Oxford University Press.

Demby, A. (1997). Algebraic procedures used by 13-to-15-year-olds. Educational Studies in Mathematics, 33, 45-70.

Filloy, E., \& Rojano, T. (1989). Solving equation: the transition from arithmetic to algebra. For the learning of mathematics, 9(2), 19-25.

Gray, E., \& Tall, D. (1994). Duality, ambiguity and flexibility: a proceptual view of simple arithmetic. Journal for Research in Mathematics Education, 26, 115-141.

Hall, R. D. G. (2002a). An analysis of errors made in the solution of simple linear equation. www.people.ex.ac.uk/PErnest/pome15/r_hall_expressions.pdf

Hall, R. D. G. (2002b). Thought processes during simplification of an algebraic expression. www.people.ex.ac.uk/PErnest/pome15/r_hall_expressions.pdf

Hart, K.M., et al. (1981). Children's understanding of mathematics: 11-16. London: John Murray.

Herscovics, N., \& Linchevski, L. (1994). A cognitive gap between arithmetic and algebra. Educational Studies in Mathematics, 27, 59-78.

Kieran, C. (1979). Children's operational thinking within the context of bracketing and the order of operations. In D. Tall (Ed.), Proceedings of the third international conference for the psychology of Mathematics Education (pp.128-135). Coventry, UK: Warwick University, Mathematics Education Research Centre.

Kieran, C. (1997). Mathematical concepts at the secondary school level: The learning of algebra and functions. In T. Nunes, \& P. Byrant, (Eds.), Learning and teaching mathematics: an international perspective (pp.133-158). United Kingdom: Psychology Press.

Kuchemann, D. E.(1981). Cognitive Demand of Secondary School mathematic Items. Educational Studies in Mathematics, 12, 301-316.

Liebenberg, R. (1997). The usefulness of an intensive diagnostic test. In P. Kelsall \& M. de Villiers (Eds.), Proceedings of the Third National Congress of the Association for Mathematics Education of South Africa: Vol. 2 (pp.72-79). Durban: Natal University.

Loh, W. F. (1991). Secondary school students' understanding of algebraic expression and equations. Unpublished master's thesis, National University of Singapore, Singapore. 
Macgregor, M., \& Stacey, K. (1997). Students understanding of algebraic notation: 11-15. Educational Studies in Mathematics, 33, 1-19.

Mayer, R. E. (1982). Different problem-solving strategy for algebraic word and equation problem. Journal of experimental and psychological: learning memory and cognition, 8, 448-462.

Ministry of Education Malaysia (1994). SPM PerfomanceReport 1994.

Ministry of Education Malaysia (1995). PMR PerfomanceReport 1995.

Ministry of Education Malaysia (1996). PMR PerfomanceReport 1996.

Ministry of Education Malaysia (2000). The Third International Mathematics and Science Study-Repeat.

Ministry of Education Malaysia (2003). Integrated Curriculum for Secondary School Syllabus Mathematic

Mulhern, G. (1989). Between the ears: making inferences about internal processes. In B. Greer, \& G. Mulhern, G. (Eds.). New directions in mathematics education (29-62). London: Routledge.

National Council of Teachers of Mathematics. (2000). Principles and standards for school mathematics. Reston: VA: National Council of Teaches of Mathematics.

Piaget, J. (1970). The science of education and the psychology of the child. New York: Grossman.

Pimm, D. (1995). Symbols and meaning in school mathematics. London: Routledge.

Radatz, H. (1979). Error analysis in mathematics education. Journal for Research in Mathematics Education, 10, 163-172.

Radford, L. (2000). Students' processes of symbolizing in algebra: a semiotic analysis of the production of signs in generalizing tasks. In Tadao Nakahara \& Masataka Koyama (Eds.), Proceedings of the $24^{\text {th }}$ conference of the International Group of the psychology of Mathematics Education Vol 4 (81-88). Hirishima, Japan

Sakpakornkan, N., \& Harries, T. (2003). Pupils' processes of thinking: Learning to solve algebraic problems in England and Thailand. In Williams, J. (Ed.), Proceeding of the British society for research into learning mathematics, 23, 91-97.

Sfard, A., \& Linchevski L. (1994). The gains and the pitfalls of reification- The case of algebra Educational Studies in Mathematics, 26, 191-228.

Swafford, J. O. \& Brown. C. A. (1989). Variables and relations: Results from the Fourth Mathematics Assessment of the National Assessment of Educational Progress. In M. M. 
Lindquist (Ed). Principles and standards for School mathematics Reston: VA: National Council of Teachers of Mathematics.

Teng, S. L. (2002). Konsepsi alternatif dalam persamaan linear di kalangan pelajar Tingkatan 4. Unpublished master's project paper, Universiti Sains Malaysia, Pulau Pinang, Malaysia.

Tirosh, D., Even, R., \& Robinson, N. (1998) Simplifying algebraic expressions: teacher awareness and teaching approaches. Educational Studies in Mathematics, 35, 51-64.

Vaiyavutjamai, P. (2001). Middle school students' performance on standard algebra tasks. In Clements, M. A., Tairab, H. H. \& Wong, K. Y. (Eds.), Energizing science, mathematics and technical education for all (260-269). Darussalam :University of Brunei.

Vlassis, J. (2002). About the flexibility of the minus sign in solving equations. In Cockburn, A. D., \& Nardi, E. (Eds.), Proceedings of the $26^{\text {th }}$ conference of the International Group of the psychology of Mathematics Education: Vol. 4. (pp. 321-328). Norwich, UK: University of East Anglia.

Warren, E. (2003). The role of arithmetic structure in the transition from arithmetic to algebra. Mathematics Education Research Journal, 15, 122-137. 